\title{
Simulation of Post-ADC Digital Beam-Forming for Large Area Radar Receiver Arrays
}

\author{
Gustav Stenberg, Johan Borg, Jonny Johansson and Gudmund Wannberg
}

\begin{abstract}
In order to provide instantaneous three-dimensional radar measurements spanning the entire vertical extent of the ionosphere, the planned EISCAT_3D incoherent scatter radar system includes multiple receive-only antenna arrays, situated at 90-280 km from the main transmit/receive site. These will employ band-pass sampling at $\sim 80 \mathrm{MHz}$, with the input signal spectrum contained in the $6^{\text {th }}$ Nyqvist zone.

This paper presents simulations and methods used to investigate use of a postADC fractional-sample-delay (FSD) system necessary to perform true time-delay beamforming. To test the feasibility and limitations of the system an extensive simulation tool has been developed. The simulation system is implemented in matlab to provide cross-platform compatibility and can be applied to any similar system. Performance degrading aspects such as noise, jitter, bandwidth and resolution can be included in the simulations.

The use of FIR-filters in the base-band of a band-pass sampled signal to apply true time-delay beam-forming is shown to be feasible.
\end{abstract}

\section{Introduction}

The next generation of incoherent scatter radars (ISRs) is going to be phased array radars. Antenna arrays provide improved capabilities to the scientific community in form of better resolution, both in space and time, and the capability to observe multiple volumes of the ionosphere simultaneously with instantaneous steering (compared to the existing EISCAT [1] antennas which are limited to $\left.3^{\circ} / \mathrm{min}\right)$. The latter will be a significant improvement to the more or less static observations of the current radars, and will allow the dynamic behavior of the ionosphere to be studied.

Atmospheric radars often use incoherent scatter techniques, with extremely weak backscattered signals at the receiving sites, often well below the background noise. Narrow beams necessary to get good spatial resolution require large antenna apertures, up to

\footnotetext{
${ }^{\circ}$ Copyright 2006 IEEE. Published in the 2006 International RF and Microwave Conference (RFM06), scheduled for September 12-14th, 2006 in Putra Jaya, Malaysia. Personal use of this material is permitted. However, permission to reprint/republish this material for advertising or promotional purposes or for creating new collective works for resale or redistribution to servers or lists, or to reuse any copyrighted component of this work in other works, must be obtained from the IEEE. Contact: Manager, Copyrights and Permissions / IEEE Service Center / 445 Hoes Lane / P.O. Box 1331 / Piscataway, NJ 08855-1331, USA. Telephone: + Intl. 908-562-3966.
} 
over 100 meters, which limit the design choice to antenna arrays if any dynamic steering capability is desired.

Because of the large extent of the ionosphere, back-scatter from different altitudes can interfere with each other, and the use of coding techniques are implemented to provide means of correlation to distinguish the signal from different altitudes. The length of the chips in the coding limits the lowest possible altitude to study since the radar cannot send and receive simultaneously.

The capabilities of the currently active and planned ISRs are summarized in Robinson [2]. The main benefit of the EISCAT_3D ISR [3] compared to others is the use of direct sampling at each antenna element, providing improved dynamic steerability and increased experimental versatility. The latter is important since the ISR research area is continuously developing new techniques and uses to improve our understanding of the ionosphere.

The EISCAT_3D ISR will be constituted by one send/receive site and multiple receive only sites, just as the existing EISCAT system is today with the aim that every limitation should be reduced by a factor of ten.

The beam-forming will be digital and centralized on a per site basis in FPGAs, i.e. data from every antenna element will be collected centrally before beam-forming. Aside from increasing the capabilities to extend the system with additional beam-formers in the future, this design is beneficial for emerging techniques like interferometry [4] since large sets of independent baselines can easily be extracted in the center.

This paper presents simulations and methods used to investigate the feasibility of using a post-ADC fractional-sample-delay (FSD) system to meet the beam-forming requirements for the EISCAT_3D system and to find the critical design aspects.

As the design develops, pre-defined and subsequent demands are collected;

- Beam-width $<0.06^{\circ}$

$\Rightarrow$ Antenna Aperture $>8.000 \mathrm{~m}^{2}$

- Beam-granularity $<0.06^{\circ}$

$\Rightarrow$ Minimum fractional delay $<15$ ps

- Lower height limit $=80 \mathrm{~km}$

$\Rightarrow$ Pulse length $\geq 200$ ns

- Dynamic range of in-band interference $\geq 60 \mathrm{~dB}$

Dynamic range of signal $\geq 24 d B$

$\Rightarrow A D C$ resolution $\geq 14$ bit

- Beamform losses $<0.1 \mathrm{~dB}$

$\Rightarrow$ ADC timing jitter $<100$ ps

- Multiple steerable beams

- Signal bandwidth $= \pm 15 \mathrm{MHz}$

- $210 \mathrm{MHz}<$ Center frequency $<240 \mathrm{MHz}$ 
The remainder of this paper will go through the design choices made, FSD FIR-filter design and optimization, and the design of a matlab system simulation software to test the filters and the system.

\section{Design Choices}

During the initial design phase, a number of choices for the EISCAT_3D system were made. To reduce the number of antenna elements in the array and to add gain for each antenna element, Yagi antennas with dual-polarization channels were chosen. Because of cross-coupling between elements the antennas will be placed sparsely in the array, at least $1.25 \lambda$ apart, causing the array to be spatially under-sampled. This introduces grating lobes in the array, which are prevented by the limited aperture of the Yagi elements.

There are two viable options on how to do beam-forming. One is phase-delay, where the signal from each antenna element is delayed up to one period, $2 \pi$, to match the phase of all elements before summing the signal. The second method is true time-delay, where the signal from the elements is delayed in time so that the arrival of the signal to each element is matched before summation. The main drawback is that the maximum delay length now is decided by the size of the array rather than the carrier wavelength.

The demands of very short pulse lengths in the EISCAT_3D system and the large antenna aperture make the use of phase-delay beam-forming impractical, since the whole array not will be illuminated simultaneously. Thus the choice was made to use true time-delay beam-forming for EISCAT_3D.

True time-delay beam-forming would cause analog delay lines to be at least as long as the array. The construction of thousands of analog delay lines with electrical lengths of $100 \mathrm{~m}$ or more is not feasible in our system. Instead, centralized post-ADC beam-forming through delay-sum steering was chosen.

The use of band-pass sampling [5] allows us to reproduce our $30 \mathrm{MHz}$ wide band in the base-band by the use of a sampling frequency of $80 \mathrm{MHz}$, which gives us a margin of $5 \mathrm{MHz}$ at each end of the band for imperfections in the anti-aliasing filters used to select the band of interest. One large question in this design choice was if digital beam-forming would be possible in this band-pass sampled system, and if so, how effective it is.

To achieve the demanded granularity of the beam-steering, the minimum step size of the delay for each element must be below 15 ps. For a 100-by-100 element array with an inter-element distance of $1.68 \mathrm{~m}$, the total delay can be as high as $550 \mathrm{~ns}$, yielding $\sim 45.000$ different delays. With an $80 \mathrm{MHz}$ sampling frequency, every multiple of $12.5 \mathrm{~ns}$ is easily handled with integer sample delays, e.g. memory buffers, which leave the fractional samples to be handled by the FSD. Applying the delays in the base-band yields that $1 / 1024^{\text {th }}$ of a sample period meets the criteria set above.

The choice for FSD fell on the application of FIR-filters. They are easy to design, characterize and implement in hardware, and have proven their worth before [6]. Using a FPGA implementation also allows for future use of IIR-filters if desired. FPGAs are available today with 18-bit hardware multipliers, giving a natural limit of 18-bit resolution to the filter coefficients. 
While deducing time-demands down to ps-levels, the question of accuracy in the timing of the array arises. Ultimately, all of the Sample\&Hold $(\mathrm{S} \& \mathrm{H})$ circuits in the entire array would open and close at the exact same time. In reality, a distributed reference clock for an array of our size will have delays in the clock distributing lines in the order of hundreds of ns, which makes it necessary to implement delay estimation techniques to achieve sub-nanosecond clock accuracy. There are ways to create accurate clock reference systems [7], but the necessary accuracy of the timing system needs to be investigated.

To test the system before prototyping and to provide a tool to validate measurements and tests, a simulation system in matlab was developed. The simulation tools will be used to design, test and improve the system before and during prototyping.

\section{$3 \quad$ Fractional Delay Beamsteering}

The use of FIR filters as an FSD in the base-band put critical demands on the filters. The filters are not allowed to introduce any phase-delays between frequencies in our signal band since that would cause parts of the summing to be destructive. Additional difficulties are introduced because of the bandwidth of our signal; $30 \mathrm{MHz}$ with a sample frequency $80 \mathrm{MHz}$ puts us close to the Nyquist limits.

There are three main design criteria to consider when designing a time-delay FIR filter; group delay error, phase delay error and amplitude error.

- The group delay error is very important since creating a group delay is what we are trying to achieve. Thus any error in the group delay reduces the delay accuracy from each antenna element, causing less than optimal beam-forming. The design criteria for the beam-forming granularity sets the minimum fractional delay; $1 / 1024^{\text {th }}$ of 12.5 ns yields delay granularity of $\sim 12.2 \mathrm{ps}$. From this we set a limit of $\sim 10 \mathrm{ps}$, in maximum total delay error. This error limit has to be met in terms of group delay, phase delay and sampling time-jitter.

- The phase delay error is of equal importance since phase errors can cause destructive interference rather than constructive. Commercially available oscillators are capable of errors $\leq 0.2 \mathrm{ps}[8]$, leaving $\sim 9.8$ ps to the filter design. Since both group delay error and phase delay error are of equal importance, they share this maximum error criteria.

- The amplitude error is only of relative importance as an error in amplitude will affect both the signal and the noise equally, with exception of thermal noise arising after the anti-aliasing filters. The amplitude error will primarily affect the beamwidth, but even a few percent of amplitude error will cause only limited beamwidening. Awaiting more specific simulation results, we assume that an amplitude error $\leq 0.1 \%$ is sufficient.

The demands on the FIR-filter design are summarized in Table 2.1. 
Table 2.1: Summary of FIR-filter design criteria

\begin{tabular}{c||c}
\hline Criteria & Limit \\
\hline \hline Group Delay Error & $\sum \leq 9.8 \mathrm{ps}$ \\
Phase Delay Error & $\leq 0.1 \%$ \\
Amplitude Error & $\leq 0$. \\
\hline
\end{tabular}
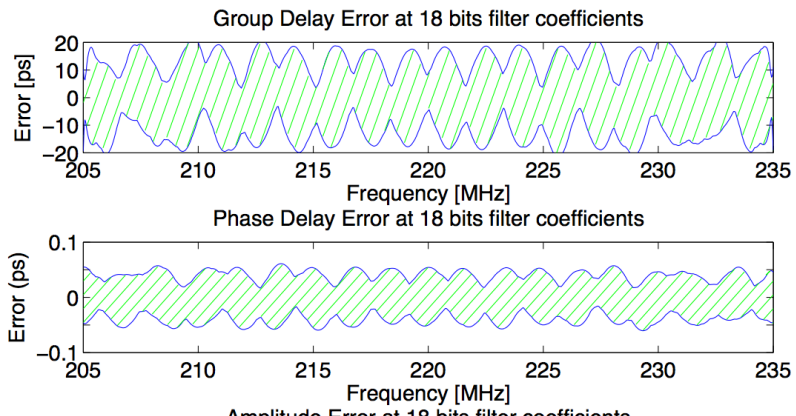

Amplitude Error at 18 bits filter coefficients

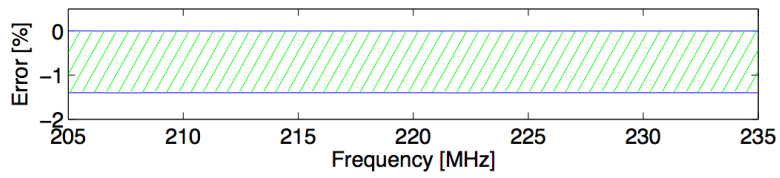

Figure 1: Max-min filter response bounds of a $75 \mathrm{~dB}$ Chebyshev windowed 48-tap filter set at 18-bit coefficient resolution.

\subsection{Filter Design}

An often used method for FIR-filter design is the Lagrange interpolation, which results in an optimally flat, ripple free amplitude and phase response. While this is highly desirable in certain applications, it would result in larger filters then necessary when the required filter performance is described by max-min error bounds for group delay, phase delay and amplitude over a defined frequency band. Thus, Lagrange interpolation is too hardware intense for our application.

Instead, the FIR filters were designed by creating the ideal filter response in the Fourier domain, adding the complex conjugate to create real coefficients, inverting the response and windowing it to create the filter coefficients. Common windowing functions, such as Hamming and Chebyshev etc. were evaluated in this fashion with reasonable results for some choices, see Figure 1. However, since no window set met the specifications over the whole band, an optimization based filter generation approach was adopted.

Different lengths of the filters where tested as the number of multipliers needed in the beam-former are directly dependent on the number of taps in the filters. 48 taps were the best trade-off choice; shorter filters introduce too large errors, longer filters demand 

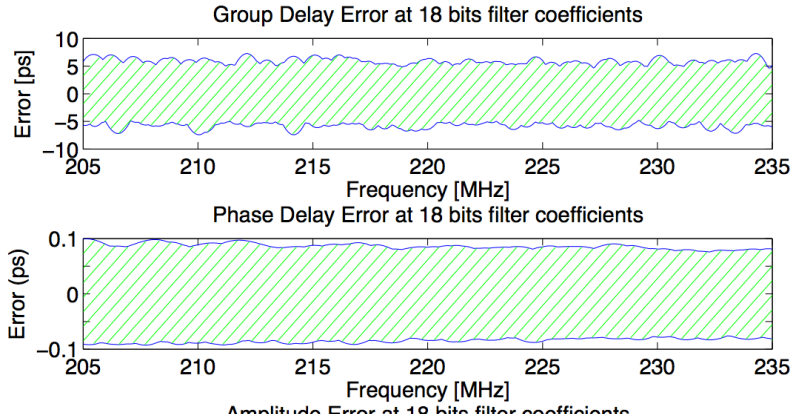

Amplitude Error at 18 bits filter coefficients

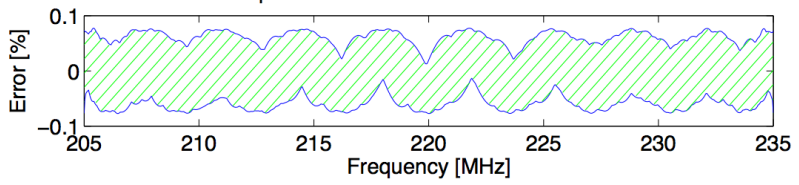

Figure 2: Max-min filter response bounds of an optimized 48-tap filter set at 18-bit coefficient resolution.

too many multipliers in realization.

\subsection{Filter Optimization}

The optimization goal was formulated as a multivariable optimization problem of minimizing the largest group delay, phase delay and amplitude error at any frequency in the band. This type of optimization problem is readily solved (without guarantees for global optimality) using fminimax (an SQP solver) in matlab. While we have not performed a study of the theoretical convergence properties of this problem, it works well enough for practical purposes. Figure 2 shows the optimized filter set responses which meet the specifications of the EISCAT_3D system.

Before implementation the filter coefficients have to be rounded from 64-bit floating point numbers to 18-bit fixed-point resolution in order to allow efficient use of the dedicated multipliers available in FPGAs. In general, the rounded coefficients will not be optimal compared to an integer search starting from the rounded optimal floating-point coefficients. Preliminary tests searching for better integer solutions have resulted in improvements to about 1 bit of coefficient precision, but this have not been applied to the filters used in this study yet.

\section{Simulation}

Matlab was chosen for the EISCAT_3D System Simulation Software as it for a multiuniversity project supplies a common programming environment available to all participants. 


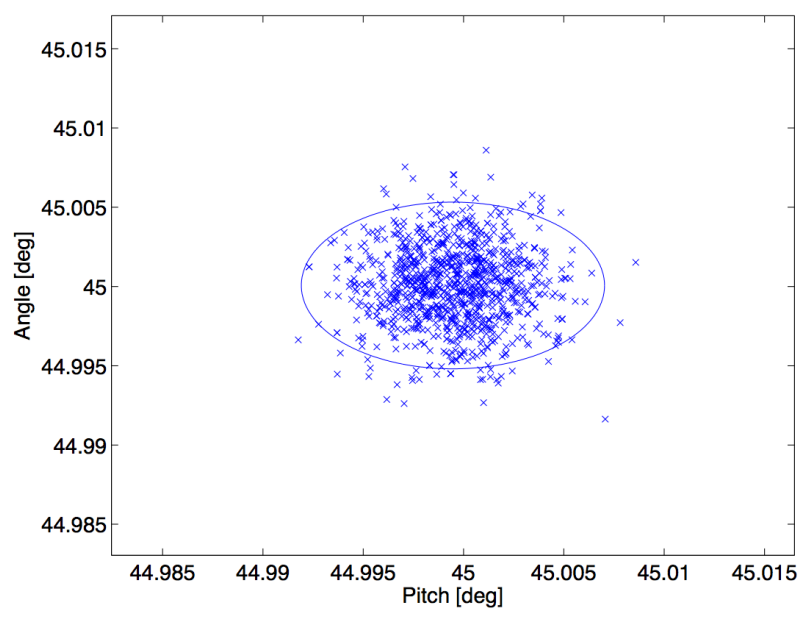

Figure 3: Pointing accuracy plot for 48-taps optimized filter set. 1000 runs, arrray 45-by-45 at jitter $100 \mathrm{ps}, 3 \sigma \approx \pm 0.0076 \mathrm{deg}$.

To have an expandable and reliable simulation tool, each part of the physical system has been designed as a matlab function so that parts can be added, updated and removed without affecting the rest of the system. The data to the array is created as analog data and then fed through each step of the receiver system, i.e. anti-aliasing filters, S\&H, ADC and beam-former. White noise is added to simulate background and temperature noise, and timing jitter is added to provide means to evaluate the effect of non-ideal timing.

With simulation of large arrays for relatively long signals, the simulation times can be large, e.g. hours to days. To provide optimization capabilities in the design steps, data from each function is stored on disk so that a change in the beam-former design does not require re-simulation of the steps before it, thus saving simulation time. The main drawback of this system is the large disk usage. With the cheap disk space available today, the space vs. time trade-off is easily made.

\subsection{Pointing \& Amplitude}

One of the main design criteria is the pointing accuracy of the beam. This aspect is mainly affected by the ability to correctly delay the signal from each antenna element, i.e. the time jitter of the system. The jitter simulations are time consuming, especially when statistical accuracy is desired. In our case, each jitter simulation consists of 1000 runs with Gaussian distributed time jitter. As can be seen in Figure 3, the pointing accuracy easily meets the $\pm 0.06^{\circ}$ criteria. Instead, the beam-forming loss criteria of $\leq 0.1 \mathrm{~dB}$ sets the maximum allowable time jitter, see Figure 4. 


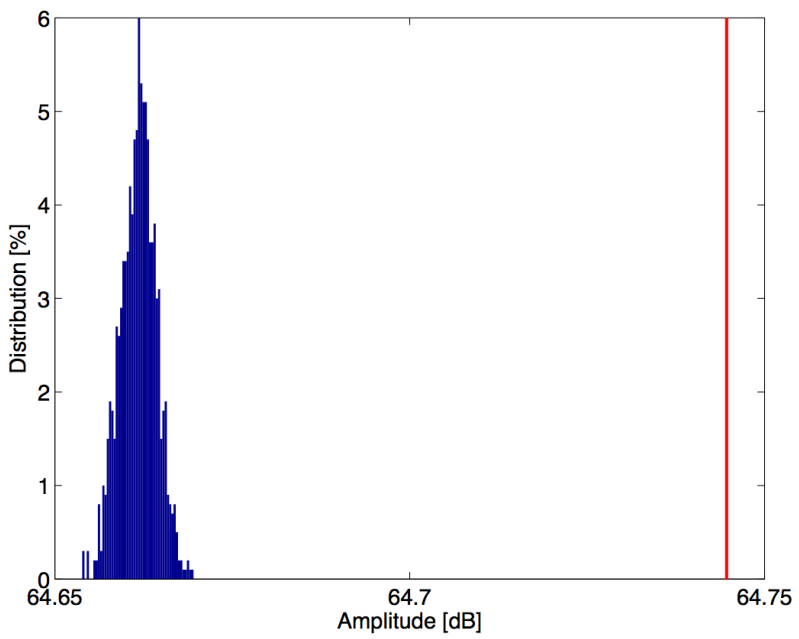

Figure 4: Amplitude accuracy plot for 48-taps optimized filter set. 1000 runs, arrray 45-by-45 at jitter $100 \mathrm{ps}, \max \approx 64.7447 \mathrm{~dB}, 3 \sigma \approx 64.6620 \mathrm{~dB}$, diff $\approx 0.0826 \mathrm{~dB}$.

\subsection{Beam Shape}

The simulations were done with $\sigma=100$ ps Gaussian distributed time jitter. 100 ps accuracy is thus good enough for the timing distribution system of EISCAT_3D as long as the jitter is Gaussian. The beam shape does not change considerably due to the Gaussian distributed time jitter, but is more prone to distortion if linear errors are introduced over the array. This stresses the point that the timing of the S\&H circuits over the whole array not only must be accurate, but any errors must be Gaussian.

\subsection{Signal Recovery}

Running a complete simulation of the system with an input signal level $32 \mathrm{~dB}$ below the noise floor, the resulting signal can still be reconstructed. Figures 5-6 shows the results after each step through the simulator, and the setup is listed in Table 2.2.

In addition to the gain achieved by beam-forming over the array, processing gain can be used to further amplify the signal, i.e. through iteration over time. The individual antenna element gain in the Yagi configuration has not been included in the current simulations. This will also increase the SNR since the Yagi antennas provide additional gain and frequency selectivity.

Figure 7 shows a comparison of the RMS values of a beam-formed coded BPSK signal with the ideal signal for a random frequency and pointing direction. Except for the exclusion of noise in the simulation, the setup is the same as in Table 2.2. As seen, the RMS value of the beam-formed signal is very close to the ideal, suggesting that the beamformer works within specifications. More work will be needed to either mathematically 
Table 2.2: Settings for 45-by-45 test array

\begin{tabular}{c||c}
\hline \multicolumn{1}{c||}{ Setting } & Value \\
\hline \hline Frequency & $217 \mathrm{MHz}$ \\
Pulse length & $200 \mathrm{~ns}$ \\
PSK \# pulses & 8 \\
PSK Code & 10101010 \\
Time jitter & $\sigma=100 \mathrm{ps}$ \\
Noise level & $-60 \mathrm{~dB}$ vs ADC range \\
Signal level & $-92 \mathrm{~dB}$ vs ADC range \\
ADC resolution & $14 \mathrm{bit}$ \\
Beam-form filter resolution & $18 \mathrm{bit}$ \\
S\&H bandwidth & $640 \mathrm{MHz}$ \\
Inter-element distance & $1.68 \mathrm{~m}$ \\
Azimuth angle of array & $45^{\circ}$ \\
Elevation angle of array & $45^{\circ}$ \\
\hline
\end{tabular}

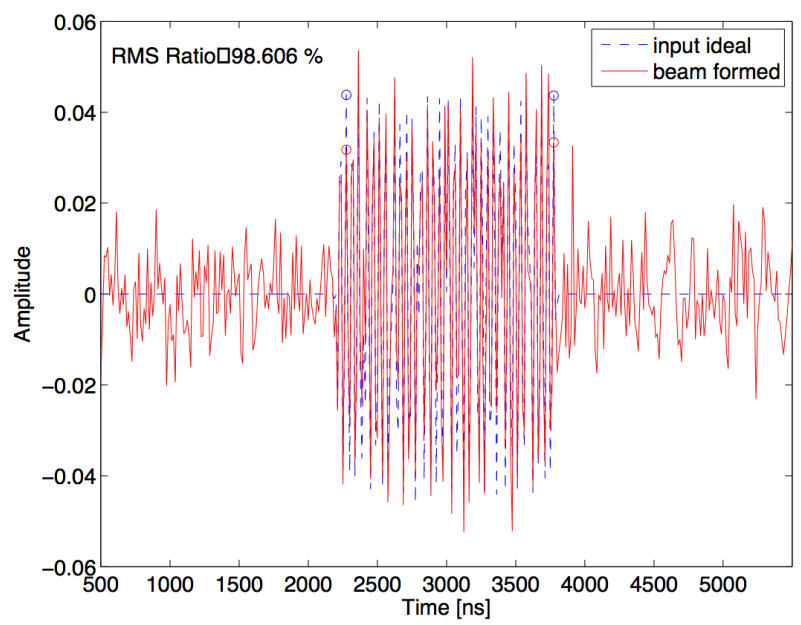

Figure 5: Comparison of beam-formed signal with ideal signal for 45-by-45 test array. 


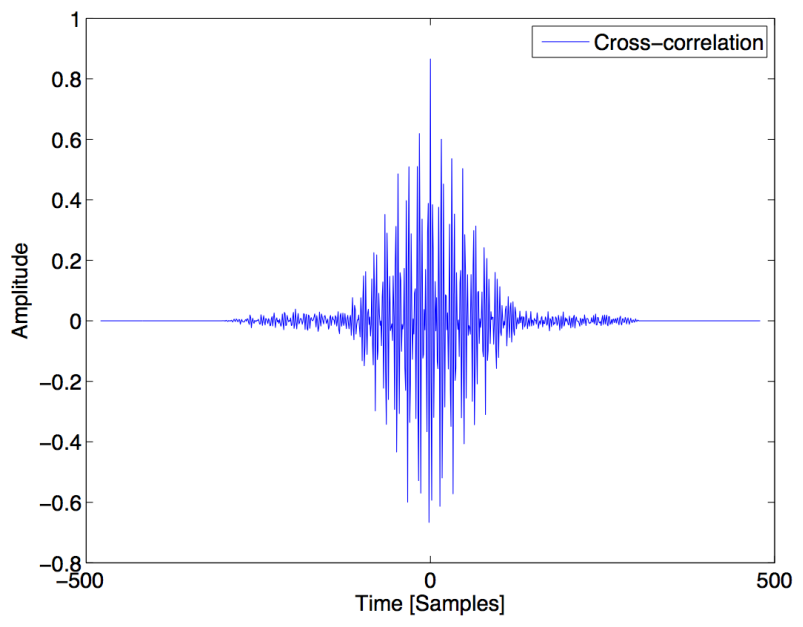

Figure 6: Cross-correlation of beam-formed signal with ideal signal for 45-by-45 test array.

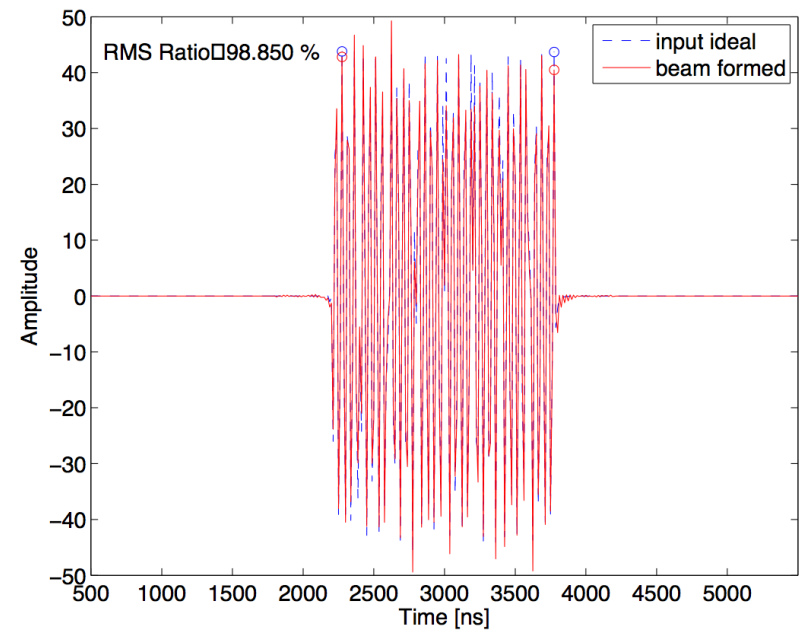

Figure 7: Comparison of beam-formed signal with ideal signal for 45-by-45 test array without noise.

or through extensive simulations show that the concept is viable for all frequencies and pointing directions specified for EISCAT_3D.

As filter lengths directly relate to the number of needed multipliers in the beamformer, optimization as to the length of the filters is highly desirable. A study is now under way to deduce the theoretical limitations of the system and to optimize the system design, e.g. filter lengths, filter coefficient resolution etc. 


\section{Conclusions}

This paper has described the evaluation of post-ADC beam-forming through true timedelay summation for large area radar receiver arrays.

The use of FIR-filter based digital beam-forming in a band-pass sampled system is found to be feasible for pulses down to $200 \mathrm{~ns}$ in length. Optimized FIR-filters with lengths of 48 taps at 18-bit coefficient resolution perform excellent over the signal-band. Reconstruction of incoming pulses $32 \mathrm{~dB}$ below the total noise floor is shown using a 45-by-45 array that spans over $74 \times 74 \mathrm{~m}$.

The maximum sampling time jitter allowed in the array is found to be $\sigma \leq 100 \mathrm{ps,}$ even though the minimum delay necessary for beam-forming is $\sim 12.2 \mathrm{ps}$, if the jitter is Gaussian.

A matlab simulation system has provided useful insight on how the EISCAT_3D system will behave and has thus clarified issues that have large impact on early design choices for the system. It will be continuously used and developed throughout the design and operation of EISCAT_3D.

\section{Acknowledgments}

The work presented in this paper was funded by the European Community under the "Structuring the European Research Area" Specific Programme Research Infrastructure action.

\section{References}

[1] G. Wannberg, I. Wolf, L.-G. Vanhainen, K. Koskenniemi, J. Rottger, M. Postila, J. Markkanen, R. Jacobsen, A. Stenberg, R. Larsen, S. Eliassen, S. Heck, and A. Huuskonen, "The EISCAT Svalbard radar: a case study in modern incoherent scatter radar system design," Radio Sci. (USA), vol. 32, no. 6, pp. 2283 - 307, November 1997. [Online]. Available: http://dx.doi.org/10.1029/97RS01803

[2] R. Robinson, "New techniques and results from incoherent scatter radars," Radio Sci. Bull. (Belgium), no. 311, pp. 79 - 94, December 2004.

[3] G. Wannberg, "EISCAT_3D design specification document," EISCAT Scientific Association, Tech. Rep., 2005. [Online]. Available: https://e7.eiscat.se/groups/ EISCAT \_3D $\$$ \% $\$ 20$ info $/ \mathrm{P} \backslash \_S \backslash \_D \backslash \_7 . p d f$

[4] T. Grydeland, C. Hoz, T. Hagfors, E. Blixt, S. Saito, A. Stomme, and A. Brekke, "Interferometric observations of filamentary structures associated with plasma instability in the auroral ionosphere," Geophys. Res. Lett. (USA), vol. 30, no. 6, pp. 71 - 1, 2003/03/15. [Online]. Available: http://dx.doi.org/10.1029/2002GL016362 
[5] C. Ackerman, C. Miller, and J. Brown, J.L., "Theoretical basis and practical implications of band-pass sampling," Proceedings of the National Electronics Conference, vol. 18, pp. $1-9,1962$.

[6] P. Murphy, A. Krukowski, and A. Tarczynski, "An efficient fractional sample delayer for digital beam steering," 1997 IEEE International Conference on Acoustics, Speech, and Signal Processing (Cat. No.97CB36052), vol. 3, pp. 2245 - 8, 1997. [Online]. Available: http://dx.doi.org/10.1109/ICASSP.1997.599498

[7] W. Grover, "A new method for clock distribution," IEEE Trans. Circuits Syst. I, Fundam. Theory Appl. (USA), vol. 41, no. 2, pp. 149 - 60, February 1994. [Online]. Available: http://dx.doi.org/10.1109/81.269051

[8] (2006, June) Data Sheet: VC-710 voltage controlled crystal oscillator. Vectron International, 267 Lowell Rd, Hudson NH 03051-4916. [Online]. Available: www.vectron.com/products/vcxo/vc710.pdf 